\title{
Characterization; Genome Sizes and Morphology of Sex Chromosomes in Hemp (Cannabis sativa L.)
}

\author{
Koichi Sakamoto ${ }^{1}$, Yukio Akiyama², Kiichi Fukui ${ }^{3}$, \\ Hiroshi Kamada ${ }^{1}$ and Shinobu Satoh ${ }^{1}$ \\ ${ }^{1}$ Institute of Biological Sciences, University of Tsukuba, Tsukuba, Ibaraki, 305-8572, Japan \\ ${ }^{2}$ Laboratory of Rice Genetic Engineering, Hokuriku National Agricultural Experiment Station, Joetsu 943-0193, Japan \\ ${ }^{3}$ Department of Biotechnology, Faculty of Engineering Graduate School of Osaka University, Suita, Osaka, 565-0871, Japan
}

Accepted October 26, 1998

\begin{abstract}
Summary A dioecious plant, Cannabis sativa has two sex chromosomes (X and $\mathrm{Y}$ ). The genome sizes of the diploid female and male plants were determined to be 1636 and $1683 \mathrm{Mbp}$, respectively, by flow cytometry. By the karyotype analysis, the $\mathrm{X}$ and $\mathrm{Y}$ chromosomes were found to be submetacentric and subtelocentric, respectively. The $\mathrm{Y}$ chromosome had the largest long arm with a satellite in the terminal of its short arm. Conspicuous condensation was specifically observed in the long arm and satellite of the $\mathrm{Y}$ chromosome during the prometaphase to metaphase stages. These results indicate that the $\mathrm{Y}$ chromosome, especially in its long arm, specifically differentiates in Cannabis sativa and might contribute to the sex determination.
\end{abstract}

The majority of flowering plants are hermaphrodites. In contrast, dioecious plants are reported to be approximately 4\% hermaphrodites among angiosperm species (Yampolsky and Yampolsky 1922). The dioecious plants, Cannabis, Rumex, and Silene reportedly have heteromorphic sex chromosomes (X and Y chromosomes; Parker 1990, Chattopadhyay and Sharma 1991) and the sex of the plants is genetically determined.

Yamada (1943) revealed that the female plants of Cannabis sativa L. have two X chromosomes whereas the male plants have one $\mathrm{X}$ and one $\mathrm{Y}$ chromosome. The $\mathrm{Y}$ chromosome is much larger than the $\mathrm{X}$ chromosome and the autosomes. The roles of the sex chromosomes in sex determination have been studied using haploid, diploid, and triploid plants of C. sativa (Warmke and Davidson 1944, Nishiyama et al. 1947). It is reported that the X and Y chromosomes of $C$. sativa carry female and male-determining genes, respectively, and that the autosomes are not involved in sex determination. There is little information, however, characterizing the sex chromosomes of $C$. sativa. In the present study, we analyzed the genome size, karyotype, and specific condensation of the $\mathrm{Y}$ chromosome of $C$. sativa to elucidate the mechanisms of sex differentiation in plants.

Materials and methods

\section{Plant materials}

The female and male plants of Cannabis sativa (family: Cannabaceae) were grown in pots at $28^{\circ} \mathrm{C}$ under a photoperiodic regime of $16 \mathrm{hr}$ of light and $8 \mathrm{hr}$ of dark. Differentiation of the female or male was determined by the flowering under a photoperiodic regime of $8 \mathrm{hr}$ of light and $16 \mathrm{hr}$ of dark for 2 weeks. The plants were vegetatively propagated by cuttings. Arabidopsis thaliana (Colombia) was grown in pots at $21^{\circ} \mathrm{C}$ under continuous light. 


\section{Estimation of nuclear genome sizes}

Nuclear genome sizes of $A$. thaliana and $C$. sativa were measured using flow cytometry. $A$. thaliana was used as the size standard. Fresh leaf samples $\left(0.5\right.$ to $\left.1 \mathrm{~cm}^{2}\right)$ were collected from mature leaves and placed on plastic petri dishes with $300 \mu \mathrm{l}$ of solution A added for nuclei isolation (High resolution DNA kit, type P: Partec GmbH, Münster, Germany). Each leaf was chopped using a sharp scalpel and remained in the solution for $5 \mathrm{~min}$. Suspensions were filtered through a $30-\mu \mathrm{m}$ nylon mesh and transferred to a sample tube. Solution B $(2 \mathrm{ml})$ was then added for DAPI staining (High resolution DNA kit, type P: Partec $\mathrm{GmbH}$ ) and they remained in the solution for $5 \mathrm{~min}$. All samples were analyzed with a Partec PA flow cytometer (Partec PA, Partec GmbH), which enabled detection of the fluorescent signals from DAPI (4',6-Diamidino-2-phenylindole Dihydrochloride). In each sample, 15000 to 35000 nuclei were analyzed at a rate of 10 to 30 nuclei/s. Peak positions and coefficients of variation $(\mathrm{CV})$ of $\mathrm{G}_{0 / 1}$ peaks were calculated using the program supplied by Partec.

\section{Preparation of the chromosomes}

Root tips (approximately $1 \mathrm{~cm}$ long) were excised and pretreated with distilled water at $11^{\circ} \mathrm{C}$ for 12 to $14 \mathrm{hr}$ and fixed with ethanol:acetic acid $(3: 1)$. Chromosome samples were prepared by the enzymatic maceration/air-drying (EMA) method (Fukui et al. 1994, Ohmido and Fukui 1996). Root tips were macerated in the enzymatic mixture ( $2 \%$ Cellulase Onozuka RS, $1.5 \%$ Pectolyase Y23, $0.3 \%$ Macerozyme R200, $1 \mathrm{mM}$ EDTA, $\mathrm{pH} 4.2$ ) at $37^{\circ} \mathrm{C}$ for 30 to $40 \mathrm{~min}$. The root tip cells were spread on glass slides and air-dried. The chromosomes from prometaphase to metaphase stages were stained with a $0.004 \%$ Giemsa solution or $7.5 \mu \mathrm{g} / \mathrm{ml}$ DAPI solution.

\section{Karyotype analysis of the chromosomes}

The chromosomal lengths were measured on a Macintosh computer using the public domain NIH image program (developed at the US National Institutes of Health and available on the Internet at http://rsb.info.nih.gov/nih-image/) and the CHIAS III system using the macro programs written by Kiichi Fukui, Seiji Kato, and Tamaki Hirose at Hokuriku National Agricultural Experiment Station, Japan (Kato et al. 1997).

\section{Results}

Distributions of the DNA content measured for the mixture of nuclei isolated from A. thaliana and female $C$. sativa or male C. sativa are shown in Figs. 1A and 1B, respectively. The peak number of 1, 2, 3, and 5 in Figs. 1A and 1B show diploid, tetraploid, octaploid and hexadecaploid $A$. thaliana, respectively as the standard. Peak number 4 shows diploid $C$. sativa. When the haploid genome size of $A$. thaliana is $130 \mathrm{Mbp}$ (Kaneko et al. 1998), the diploid genome sizes in female and male plants of C. sativa are estimated to be $1636 \pm 7.2$ (S.D.) and $1683 \pm 13.9$ (S.D.) Mbp, respectively (Table 1).

Female and male $C$. sativa have $\mathrm{XX}$ and $\mathrm{XY}$ chromosomes with 18 autosomes, respectively. Condensation patterns were observed in the Giemsa-stained female and male $C$. sativa chromosomes at the pro-metaphase stage (Figs. 2A, 2B). The Y chromosome, the largest chromosome, is subtelocentric and has a large long arm with a satellite in the terminal of its short arm. The X chromosome was found to be submetacentric with a short satellite at the end of the short arm.

Conspicuous condensation was observed over the entire long arm and satellite of the $\mathrm{Y}$ chromosome from prometephase to metaphase (Figs. 3A, 3B). Fig. 4A shows a model of the condensation pattern of the $\mathrm{Y}$ chromosome. Fig. 4B shows the dynamics and correlation of the length of the satellite, short arm and long arm against the total length of the $\mathrm{Y}$ chromosome from prometaphase to metaphase. The result indicates that the satellite and long arm of the $\mathrm{Y}$ chromosome condensed 5 

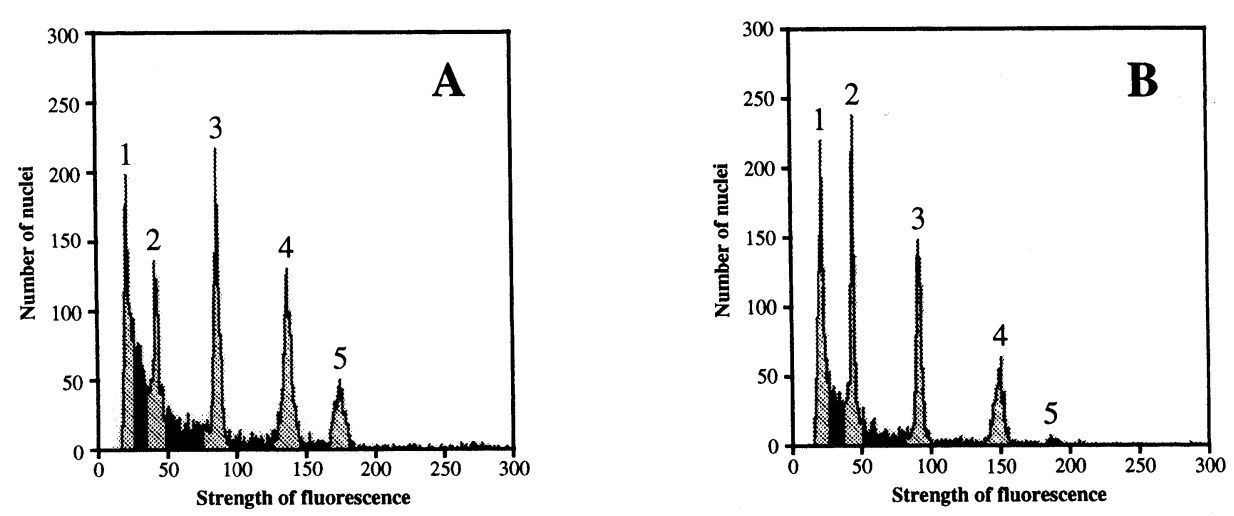

Fig. 1. Nuclear DNA content isolated from leaf tissues of $C$. sativa and A. thaliana. A) The female plant of C. sativa (peak 4, CV=1.8-2.5\%) and A. thaliana. B) The male plant of C. sativa (peak 4, $\mathrm{CV}=1.3-2.9 \%$ ) and $A$. thaliana. Peaks $1,2,3$ and 5 correspond to diploid, tetraploid, octaploid and hexadecaploid of $A$. thaliana, respectively.
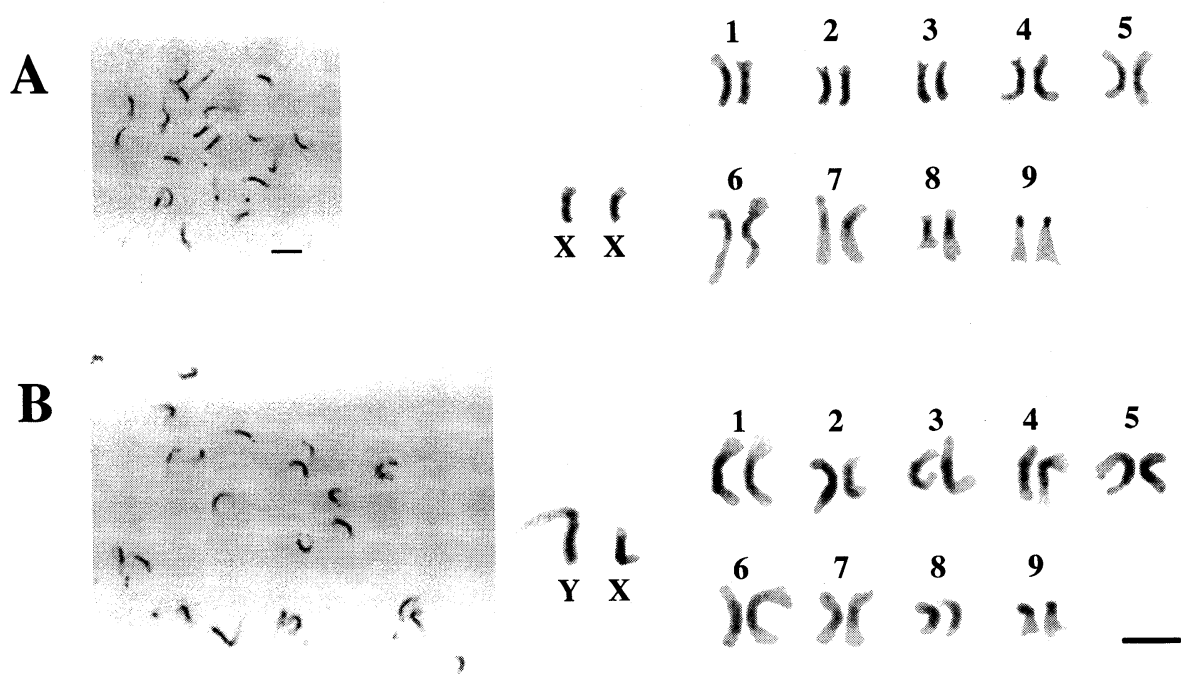

Fig. 2. Chromosomes and karyograms with $\mathrm{X}$ and $\mathrm{Y}$ chromosomes of $C$. sativa at the pro-metaphase stage. A) female. B) male. Bars indicate $5 \mu \mathrm{m}$.

and 6.5 times more than the short arm, respectively.

\section{Discussion}

Flow cytometry of the nuclear DNA content has become an important technique for detection of genome sizes of various organisms. In many plant species, young leaves are commonly used for the flow cytometry. In the present study, we used only the mature leaves of C. sativa for flow cytometry, because young leaves are more contaminated by mitotic chromosomes. Cell division is probably still active in the
Table 1. Nuclear genome size of Cannabis sativa

\begin{tabular}{cc}
\hline \hline Species & $\begin{array}{c}\text { Genome size (diploid) } \\
(\mathrm{Mbp})\end{array}$ \\
\hline $\begin{array}{c}\text { Arabidopsis thaliana } \\
\text { Cannabis sativa } \text { L. } \\
\text { female ( }(+)\end{array}$ & 260 \\
male (ơ) & $1636(7.2)$ \\
\end{tabular}

Each value ( $\pm /-$ S.D.) is the mean of results from six measurements. 

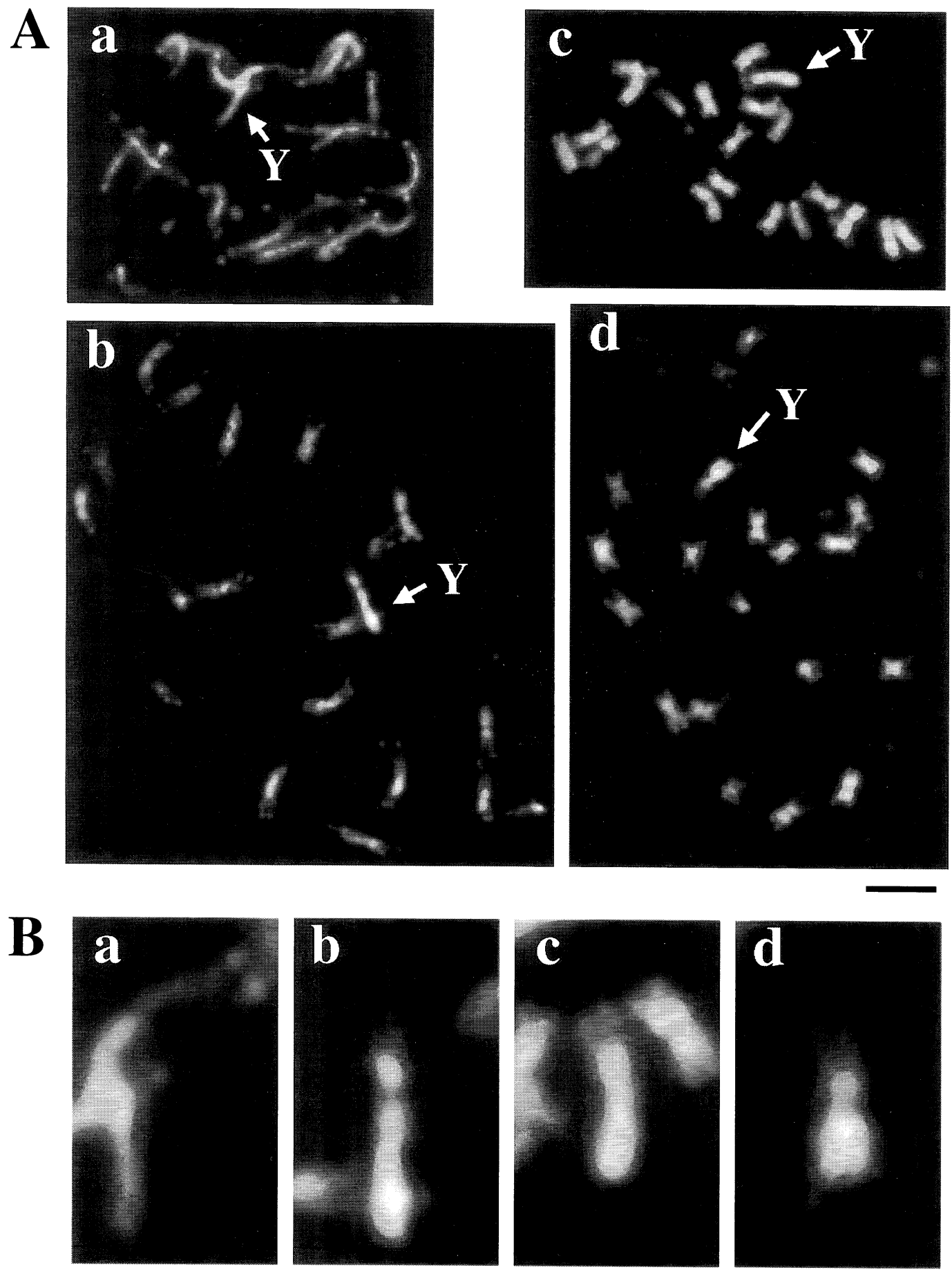

Fig. 3. Chromosomes $(2 n=20)$ at the prometaphase to metaphase stages stained with DAPI. A a-d) Arrows indicate the $\mathrm{Y}$ chromosome in the male plant. Bars indicate $5 \mu \mathrm{m}$. B a-d) are the enlarged $\mathrm{Y}$ chromosomes at each stage. 


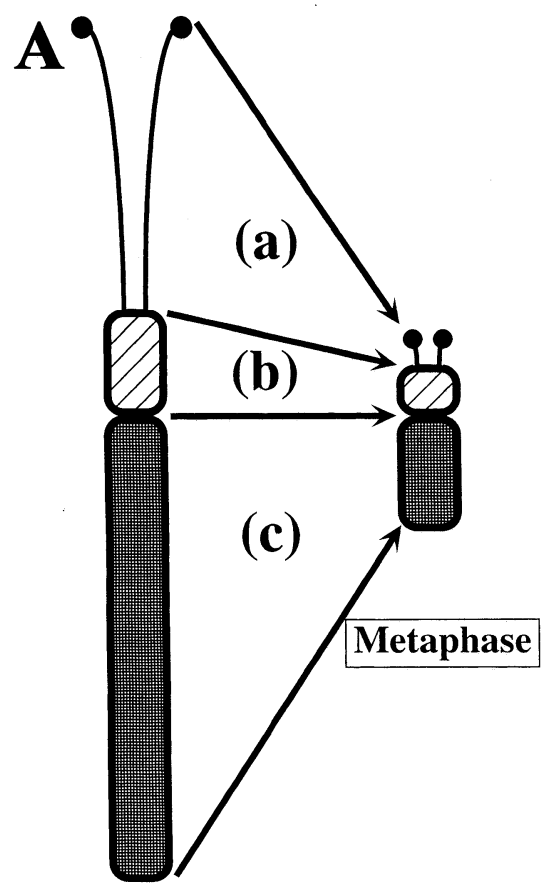

Prometaphase
B

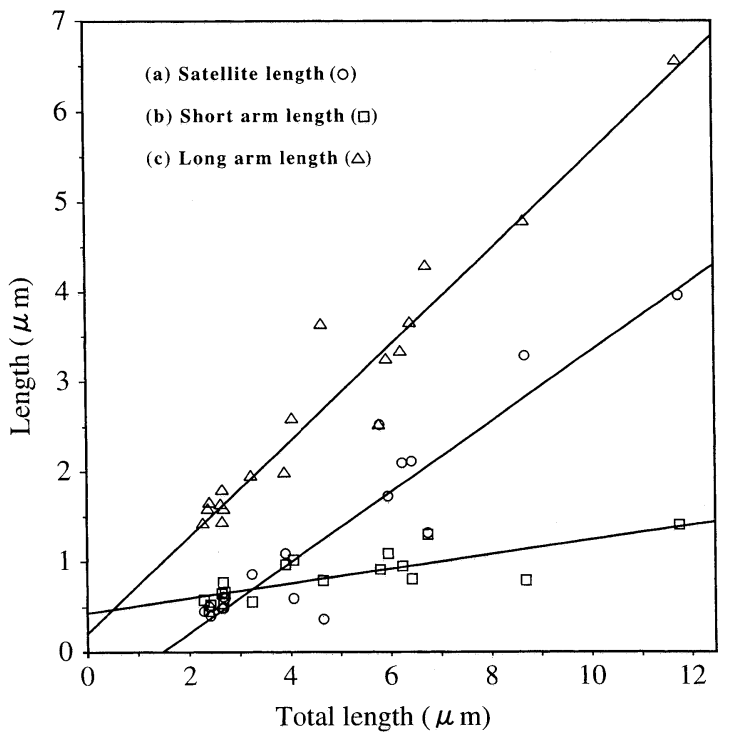

(a) Satellite length $(\mu \mathrm{m})=\mathbf{- 0 . 5 9 2 + 1 . 3 9 1 *}$ Total length $(\mu \mathrm{m}) ; \mathbf{R}^{\wedge} 2=0.87$

(b) Short arm length $(\mu \mathrm{m})=0.404+0.081 *$ Total length $(\mu \mathrm{m}) ; \mathbf{R}^{\wedge} 2=0.60$

(c) Long arm length $(\mu \mathrm{m})=0.187+0.528 *$ Total length $(\mu \mathrm{m}) ; \mathbf{R}^{\wedge} 2=0.94$

Fig. 4. Schematic illustration of specific condensation of the long arm and satellite of the Y chromosome. A) The correlation of the length of the $\mathrm{Y}$ chromosome represented graphically. B a-c) the satellite, short and long arms, respectively.

young leaves of $C$. sativa.

We detected differences in the DNA content between female and male $C$. sativa. The differences in the nuclear DNA content was approximately $47 \mathrm{Mbp}$ (Table 1). This value occupies about $2.8 \%$ of the total genome size of the male plants and could correspond to the large long arm of the $\mathrm{Y}$ chromosome. In another dioecious plant, Silene latifolia, there is a 2 to $5 \%$ difference in the nuclear DNA content between females and males (Costich et al. 1991, Vagera et al. 1994, Doležel et al. 1995). Differences might be due to the large size of the Y chromosome.

The chromosomes of $C$. sativa condensed from the mitotic prometaphase to metaphase stages in a similar manner, except for the $\mathrm{Y}$ chromosome (Figs. 3A, 3B). The satellite and long arm of the $\mathrm{Y}$ chromosome were more rapidly condensed in comparison with the short arm, $\mathrm{X}$ chromosome and autosomes. These results indicate that the Y chromosome, especially in its long arm, specifically differentiates in $C$. sativa chromosome. This region might be associated with the accumulation of specific nucleotide sequences (Sakamoto et al. in press) and differentiation of the sex chromosomes.

In order to elucidate the structure of the $\mathrm{Y}$ chromosome of $C$. sativa, we compared female and male DNAs by the random-amplified polymorphic DNA (RAPD) technique. One DNA fragment, $729 \mathrm{bp}$ in size, produced much more intense bands specific to male plants, in addition to less intense bands common to both the sexes in a gel-blot analysis of genomic DNA and was named MADC1 (male-associated DNA sequence in C. sativa) (Sakamoto et al. 1995). A fluorescence in situ hybridization (FISH) experiment (Fukui et al. 1994, Ohmido and Fukui 1996, 1997) using metaphase chromosomes from root cells with $M A D C 1$ as the probe detected a clear doublet signal only on the end of the long arm of the Y chromosome (Sakamoto et al. 1997). These results demonstrate that 
some specific DNA sequences might be accumulated and associated with differentiation and functions of the sex chromosome, especially in the long arm of the Y chromosome. Further investigation of the molecular structure of the sex chromosomes will contribute to the elucidation of mechanisms of sex differentiation and heteromorphism of sex chromosomes in plants.

\section{Acknowledgment}

We thank Ms. R. Ishizuka and Mr. J. Iijima (Ikeda Scientific Co., Ltd.) for assistance with the flow cytometric analysis, and Ms. M. Ikeda (Institute of Biological Sciences, University of Tsukuba) for her generous gift of $A$. thaliana.

This study was supported in part by a Grant-in-Aid for Special Research on Priority Areas (07281101, Genetic Dissection of Sexual Differentiation and Pollination Process in Higher Plants) from the Ministry of Education, Science, Culture and Sports, Japan.

\section{References}

Chattopadhyay, D. and Sharma, A. K. 1991. Sex determination in dioecious species of plants. Federation Proc. 102: $29-55$.

Costich, D. E., Meagher, T. R. and Yurkow, E. J. 1991. A rapid means of sex identification in Silene latifolia by use of flow cytometry. Plant Mol. Biol. Rep. 9: 359-370.

Doležel, J. and Göhde, W. 1995. Sex determination in dioecious plants Melandrium album and M. rubrum using high-resolution flow cytometry. Cytometry 19: 103-106.

Fukui, K., Ohmido, N. and Khush, G. S. 1994. Variability in rDNA loci in genus Oriza detected through fluorescence in situ hybridization. Theor. Appl. Genet. 87: 893-899.

Kaneko, T., Kotani, H., Nakamura, Y., Sato, S., Asamizu, E., Miyajima, N. and Tabata, S. 1998. Structural analysis of Arabidopsis thaliana chromosome 5 . V. Sequence features of the regions of $1,381,565$ bp covered by twenty one physically assigned P1 and TAC clones. DNA Res. 5: 131-145.

Kato, S., Hirose, T., Akiyama, Y., O’Neill, C. M. and Fukui, K. 1997. Manual on the chromosome image analyzing system III, CHIAS III. Res. Rep. Agricul. Develop. Hokuriku Area. 36: 1-76.

Nishiyama, I., Yamada, I. and Mazaki, M. 1947. Studies on artificial polyploid plants. XI. Changes of the sex ratio in the progeny of the autotetraploid hemp. Seiken Ziho 3: 144-151.

Ohmido, N. and Fukui, K. 1996. A new manual for fluorescence in situ hybridization (FISH) in plant chromosomes. Rice Genet. Newsl. 13: 89-93.

- and - 1997. Visual verification of close disposition between a rice A genome-specific DNA sequence (TrsA) and the telomere sequence. Plant Mol. Biol. 35: 963-968.

Parker, J. S. 1990. Sex-chromosome and sexual differentiation in flowering plants. Chromosomes Today 10: 187-198.

Sakamoto, K., Shimomura, K., Komeda, Y., Kamada, H. and Satoh, S. 1995. A male-associated DNA sequence in a dioecious plant, Cannabis sativa L. Plant Cell Physiol. 36: 1549-1554.

-, - - - Fukui, K., Kamada, H. and Satoh, S. 1997. analysis of the structure of sex chromosomes in a dioecious plant, Cannabis sativa L. Plant Physiol. 114(Suppl.): 243.

Vagera, J., Paulíková, D. and Dolezel, J. 1994. The development of male and female regenerants by in vitro androgenesis in dioecious plant Melandrium album. Ann. Bot. 73: 455-459.

Warmke, H. E. and Davidson, H. 1944. Polyploid investigation. Year book of the Carnegie Instit. of Washignton. 43: 135139.

Yamada, I. 1943. The sex-chromosome of Cannabis sativa L. Seiken Ziho 2: 64-68.

Yampolsky, C. and Yampolsky, H. 1922. Distribution of the sex forms in the phanerogamic flora. Bibl. Genet. 3: 1-62. 